Rev. Int. Contam. Ambie. 34 (3) 453-465, 2018

DOI: 10.20937/RICA.2018.34.03.08

\title{
EVALUACIÓN DE UN PROCESO INDUSTRIAL DE PRODUCCIÓN DE BIODIÉSEL MEDIANTE ANÁLISIS DE CICLO DE VIDA
}

\author{
Salvador CARLOS HERNÁNDEZ* y María de Lourdes Virginia DÍAZ JIMÉNEZ
}

Centro de Investigación y de Estudios Avanzados del Instituto Politécnico Nacional, Unidad Saltillo. Avenida Industria Metalúrgica 1062, Parque Industrial Ramos Arizpe, Ramos Arizpe, Coahuila, México, C. P. 25900

*Autor para correspondencia: salvador.carlos@cinvestav.mx

(Recibido marzo 2017; aceptado octubre 2017)

Palabras clave: impacto ambiental, biocombustible, grasa residual

\section{RESUMEN}

En este documento se presenta un análisis de ciclo de vida de un proceso de producción de biodiésel a partir de grasas residuales vegetales. El objetivo fue evaluar el desempeño ambiental del proceso e identificar oportunidades de mejora. Las grasas provienen de la industria restaurantera de la zona metropolitana de Tijuana, Baja California, México y son procesadas por la empresa Enremex S. de R. L. de C. V. Se emplea la reacción de transesterificación con catalizador homogéneo para producir cada año $1200000 \mathrm{~L}$ de biodiésel y aproximadamente $240000 \mathrm{~L}$ de glicerol crudo. Como alternativa al catalizador homogéneo, se propone la aplicación de un catalizador heterogéneo basado en una zeolita natural. Para lo cual se realizaron experimentos a nivel laboratorio. Respecto a la evaluación del impacto ambiental, el proceso se dividió en tres etapas: a) recolección de materia prima, b) producción de biodiésel y c) manejo de residuos. Asimismo, se consideraron dos escenarios: 1) transesterificación con catalizador homogéneo, 2) transesterificación con catalizador heterogéneo. Los resultados indican que la etapa de producción tiene la mayor contribución en el impacto global del proceso. Asimismo, el uso del catalizador heterogéneo disminuye en gran medida el impacto ambiental negativo en comparación con el catalizador homogéneo.

Key words: environmental impact, biofuel, waste fat

\begin{abstract}
This document focuses on a life cycle assessment of a biodiesel production process from vegetal waste fats. The goal was to evaluate the environmental performance of the process and to identify opportunities to improve it. Those wastes are originated by the restaurant industry on the urban zone of Tijuana, Mexico and they are processed by Enremex S. de R. L. de C. V. A transesterification reaction with homogeneous catalyst is used to produce $1200000 \mathrm{~L}$ of biodiesel and around $240000 \mathrm{~L}$ of glycerol per year. As an alternative to the homogeneous catalyst, a based natural zeolite heterogeneous catalyst is proposed. Then, a series of laboratory experiments were performed. Concerning the environmental assessment, the biodiesel production process was separated in three steps: a) raw material collection, b) biodiesel production, and c) wastes management. In addition, two scenarios were considered: 1) transesterification with homogeneous catalyst,
\end{abstract}


2) transesterification with heterogeneous catalyst. The results show that the production stage is the one that contributes the most on the environmental impact. Also, the use of a heterogeneous catalyst reduces the environmental impact in comparison with the homogeneous one.

\section{INTRODUCCIÓN}

\section{Biodiésel a partir de grasas residuales}

La producción de aceites y grasas residuales es elevada en todas las áreas metropolitanas del país y con frecuencia representa problemas técnicos, económicos y ambientales. Por ejemplo, su disposición sin tratamiento en las redes de alcantarillado puede causar bloqueos en las líneas de conducción, corrosión de tuberías y malos olores, entre otras. También, afectan la eficiencia de las plantas de tratamiento de aguas residuales, puesto que requieren procesos específicos para su eliminación (Tacias Pascacio et al. 2016, Wallace et al. 2017).

Para el manejo y control de estos residuos se han establecido acciones diversas, las cuales van desde la concientización para reducir su producción, hasta el desarrollo de procesos para su revaloración (Wallace et al. 2017). Entre estos últimos, la producción de biodiésel es considerada una de las alternativas con mayores ventajas (Mandolesi de Araújo et al. 2013, Talebian-Kiakalaieh et al. 2013, Yaakob et al. 2013, Hajjari et al. 2017). En efecto, este biocombustible presenta características técnicas favorables para su aplicación en motores diésel. Además, su producción ha demostrado ser rentable. No obstante, la producción de biodiésel a partir de grasas residuales está condicionada por la calidad de la materia prima.

La grasa amarilla (aceite vegetal residual producido en la cocción de alimentos) contiene impurezas que deben ser retiradas antes de la transesterificación. También pueden contener un alto porcentaje de ácidos grasos libres, lo que conduce a un requerimiento mayor de metanol para evitar la formación de jabones.

La grasa café (obtenida de trampas de grasa) contiene una concentración elevada de materia orgánica, lo que provoca malos olores y presenta altos porcentajes de ácidos grasos libres que pueden hacer inviable la transesterificación directa. Todo lo anterior implica un pretratamiento mayor que aumenta los costos de producción.

En cuanto al aspecto ambiental, es importante identificar ventajas y desventajas, así como estrategias para minimizar eventuales riesgos. En este sentido, el análisis de ciclo de vida (ACV) es un método útil para este efecto, por lo que ha sido aplicado en diversos estudios (Talens Peiró et al. 2010, Yano et al. 2015, Caldeira et al. 2016, Siegel Moecke et al. 2016, Lombardi et al. 2018).

\section{Aplicación del ACV en la obtención de biodiésel a partir de grasas residuales}

El ACV permite cuantificar y caracterizar los impactos potenciales sobre el medio ambiente asociados con un producto o un proceso durante su ciclo de vida (Guinée 2002, Muralikrishna y Manickam 2017). Este método ha sido estandarizado en las normas ISO 14040 (ISO 14040 2006, ISO 14044 2006) y se resume en la figura 1.

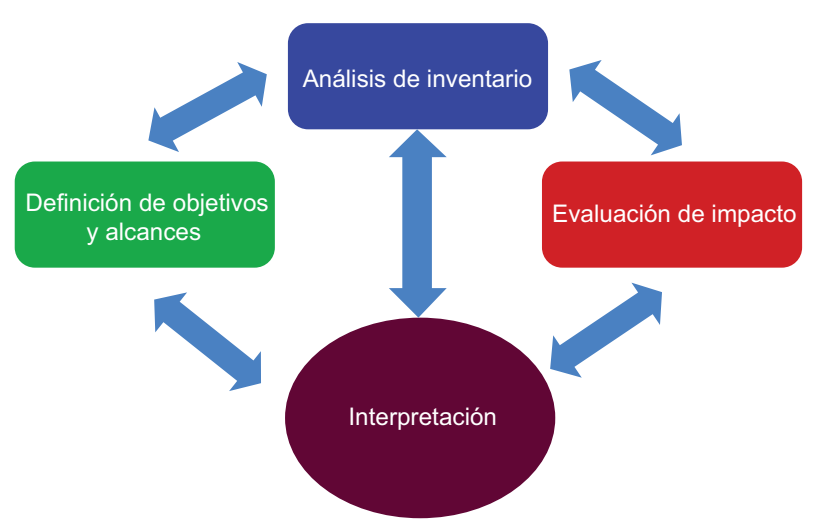

Fig. 1. Esquema general del método análisis del ciclo de vida

Entre las fortalezas de dicho método se encuentran las siguientes (Finnveden et al. 2009, Curran 2014):

- Produce un análisis completo puesto que es posible incluir todas las etapas del ciclo de vida (de la cuna a la tumba)

- Identifica áreas de oportunidad ya que considera la interacción de actividades y etapas a través de un pensamiento holístico

- Considera una amplia gama de impactos ambientales, incluyendo la comparación entre ellos aún cuando sean de naturaleza diferente

- Permite la proyección de escenarios futuros o hipotéticos para estudiar diferentes condiciones de operación 
- Promueve la comunicación entre los agentes involucrados en el ciclo de vida, con lo que se genera una mayor conciencia de las implicaciones globales de un elemento o etapa

Algunas de las limitantes son (Finnveden et al. 2009, Curran 2014):

- Se requieren muchos datos para conseguir un inventario completo ya que la falta de ellos pueden condicionar los resultados obtenidos. Por tal motivo, se sugiere complementar el inventario con datos teóricos reportados, simulaciones e interpolaciones, también se trabaja en el desarrollo de bases de datos más completas

- Algunas categorías específicas como uso de suelo, biodiversidad, recursos del agua, salud humana, presentan alto grado de dificultad, por lo que se requieren mejorar los métodos de evaluación

- Existen tres principales fuentes de incertidumbre (Guldbrandsson y Bergmark 2012): los parámetros (incertidumbre estadística de los datos), los escenarios (generada por la selección de aspectos como la asignación de cargas ambientales o los valores de corte) y los modelos (relacionada con la complejidad de los sistemas y la falta de conocimiento de ellos)

- La asignación de créditos a cambio de cargas evitadas suele emplearse como una alternativa compensatoria. Sin embargo, esto puede requerir una cantidad adicional de datos

- Con frecuencia es necesario un análisis adicional para la toma de decisiones

No obstante, el ACV es una herramienta aceptada por la comunidad científica para la evaluación de productos y procesos. Es un tópico de investigación con amplia actividad, lo cual implica que sigue evolucionando.

En el caso de la producción de biodiésel a partir de grasas residuales, se han reportado varios estudios basados en el ACV. Algunos de ellos se describen brevemente a continuación.

En una investigación se analizó la producción de $1 \mathrm{t}$ de biodiésel considerando cuatro etapas: recolección, pretratamiento, transporte y transesterificación. Los autores concluyeron que la transesterificación contribuye con $68 \%$ del impacto total. Esto se debe principalmente al uso de energía eléctrica, la cual se genera con fuentes no renovables (Talens Peiró et al. 2010).

En otro trabajo se determinó que el uso de biodiésel producido a partir de grasas residuales ofrece un menor impacto en categorías como el calentamiento global, consumo de combustibles fósiles, contaminación del aire y acidificación de suelos (Yano et al. 2015). También se ha evaluado el impacto de los métodos de recolección de grasas (contenedores, casa por casa y restaurantes). Se ha encontrado que esta etapa influye de manera considerable en el impacto global del sistema de producción de biodiésel, por lo que es importante implementar mecanismos óptimos para la recolección (Caldeira et al. 2016). En tanto que Siegel Moecke et al. (2016) reportan que en una planta de producción de 200 L por día, la recolección de materia prima es la etapa que más emisiones contaminantes genera (92\%). Mientras que la fase de producción contribuye con el $7 \%$ de emisiones.

Por otro lado, Lombardi et al. (2018) evaluaron diferentes catalizadores [hidróxido de sodio $(\mathrm{NaOH})$, hidróxido de potasio $(\mathrm{KOH})$, ácidos y fluidos supercríticos]. Concluyeron que tanto el $\mathrm{NaOH}$ como el $\mathrm{KOH}$, inducen un mejor desempeño, principalmente por su menor consumo de energía. También, se determinó que el $\mathrm{NaOH}$ resulta una mejor opción respecto al potencial de calentamiento global y al consumo acumulativo de energía.

De lo anterior se muestra que existe un interés amplio por la evaluación del desempeño ambiental de la producción de biodiésel a partir de grasas residuales. En este sentido, el ACV es una herramienta para evaluar impactos ambientales, identificar problemáticas (áreas de oportunidad) y comparar escenarios.

\section{Caso de estudio}

En este trabajo se presentan resultados de una investigación relacionada con la evaluación del impacto ambiental de un proceso industrial de producción de biodiésel. De acuerdo con la Ley General del Equilibrio Ecológico y la Protección al Ambiente (SEMARNAT 2017), el impacto ambiental se define como la modificación del ambiente ocasionada por la acción del hombre o de la naturaleza. Así se entenderá en el resto del documento.

La materia prima consiste en grasas residuales generadas por la industria restaurantera de la zona metropolitana de Tijuana, Baja California. Las grasas son procesadas por la empresa Enremex S. de R.L. de C. V. Se producen 1200000 L de biodiésel y alrededor de $240000 \mathrm{~L}$ de glicerol crudo por año.

Como una alternativa al catalizador homogéneo actualmente utilizado $(\mathrm{KOH})$, basado en una zeolita natural, la finalidad es mejorar aspectos del proceso, tales como la facilidad de separación del biodiésel y la glicerina. También se espera una disminución en la generación de agua residual y de residuos sólidos. 
En la figura 2 se presenta un esquema del proceso bajo estudio.

El desempeño del proceso actual y de la propuesta de este trabajo se evalúa a partir de un estudio de ACV. El objetivo de esta investigación es establecer ventajas y/o desventajas técnicas y ambientales del proceso de obtención de biodiésel, así como plantear estrategias para la mejora del mismo.

\section{MATERALES Y MÉTODOS}

\section{Definición de objetivos y alcances del estudio de impacto ambiental}

El objetivo de este trabajo es evaluar el desempeño, mediante el $\mathrm{ACV}$, de un proceso de producción de biodiésel a partir de aceite vegetal residual para identificar áreas de mejora. Lo que implica minimizar impactos ambientales de los dos escenarios considerados:

i. Escenario 1. Se trata de la situación actual del proceso de producción de biodiésel, donde se consideran tres etapas:

En la primera se incluye la recolección y el transporte de la materia prima. La recolección se realiza en camionetas de gasolina y se transporta a la empresa en camión de diésel. La materia prima recolectada pasa por un proceso de filtración mecánico para eliminar partículas sólidas.

En la segunda etapa se agrupan las fases de pretratamiento, transesterificación y separación de productos. El pretratamiento consiste en la separación de partículas sólidas por métodos físicos (decantación y filtración). La materia prima no es sometida a pretratamiento químico (como la esterificación) a fin de evitar el incremento en los costos de producción de biodiésel y poder ofrecer al mercado competitividad en precios. Las impurezas sólidas recuperadas, con alto contenido en proteínas, son vendidas para su procesamiento y transformación en base de alimento para animales de compañía (mascotas). La separación de productos se realiza por métodos físicos (decantación, filtración, evaporación) para obtener biodiésel, glicerol crudo, agua residual, jabones y residuos sólidos.

La tercera etapa consiste en el manejo de residuos. El agua residual y los jabones producidos se recuperan en bruto junto con el glicerol crudo. Esta mezcla se neutraliza y se envía en camión de diésel para su venta. Actualmente se buscan alternativas para la disposición del agua residual in situ. Por ejemplo: tratamiento por electrofloculación, tratamiento en humedales y su aplicación en procesos de biocomposta. Finalmente, los residuos sólidos se dejan secar y se envían para su venta en una camioneta de gasolina.

ii. Escenario 2. Se consideran las mismas tres etapas que para el primer escenario. Adicionalmente, se plantea la sustitución del catalizador homogéneo por uno heterogéneo, el cual se basa en una zeolita natural mexicana, la heulandita cálcica.

La zeolita utilizada posee una baja relación $\mathrm{Si}$ / $\mathrm{Al}=4.4$, lo que le confiere un carácter ácido. Sin embargo, su tamaño de poro es pequeño $(5.32 \mathrm{~nm})$,

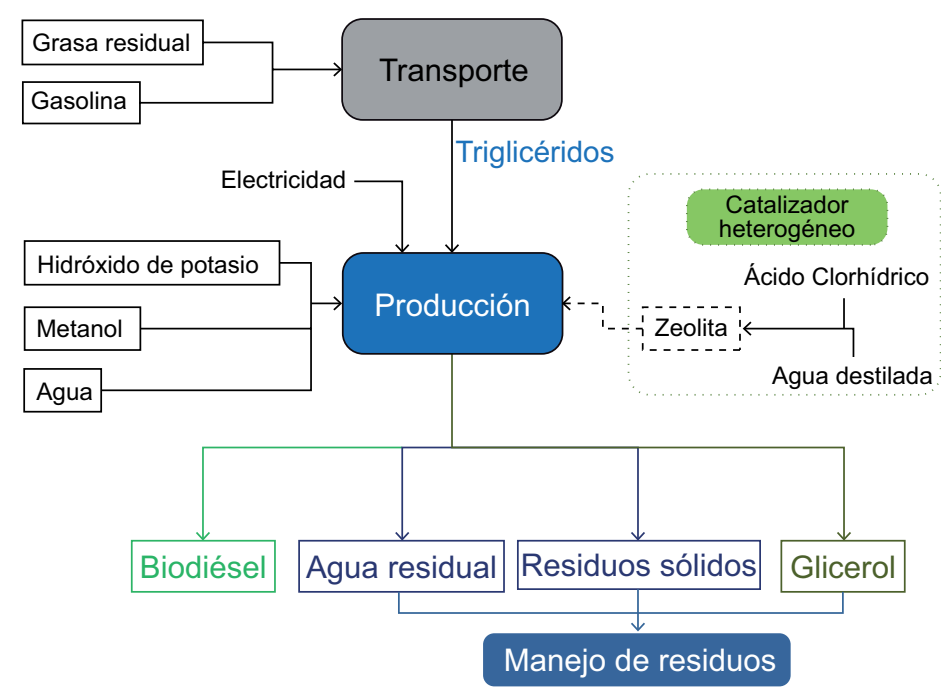

Fig. 2. Esquema del proceso de producción de biodiésel bajo estudio 
por lo que se somete a un proceso suave de desaluminación. Así, se agranda el tamaño de poro, sin reducir su acidez, y se permite un mayor acceso de las moléculas de triglicérido para conseguir su activación.

En este escenario se incluye la etapa de preparación del catalizador, la cual consiste en una desaluminación con ácido clorhídrico al 37 \% y el lavado con agua destilada. En el anexo se describe el procedimiento seguido para la realización de experimentos a nivel laboratorio.

Las ventajas de este catalizador son: permite realizar simultáneamente reacciones de esterificación y transesterificación (evita una eventual esterificación previa a la transesterificación), facilita la manipulación y recuperación del catalizador, permite obtener productos de mayor calidad (biodiésel y glicerol).

La unidad funcional para el ACV se ha establecido como el procesamiento de $1000 \mathrm{~L}$ de grasa vegetal residual.

Por otro lado, es importante señalar que no se consideran mecanismos de aprovechamiento de biodiésel, únicamente la producción del mismo con una calidad similar a la indicada por las normas de la Sociedad Americana para Pruebas y Materiales (ASTM, por sus siglas en inglés).

\section{Conformación del inventario}

La información empleada en el ACV del proceso de producción de biodiésel se divide en tres apartados:

1. Aquella proporcionada por la empresa (datos directos). Estos corresponden a las etapas de recolección de materia prima y a la de transesterificación con catalizador homogéneo.

2. La generada a nivel laboratorio (datos generados por vía experimental), la cual se relaciona con experimentos para determinar el rendimiento del catalizador heterogéneo y compararlo con el catalizador homogéneo.

3. La obtenida a partir de información documental (datos teóricos).
En el cuadro I se muestran los resultados del análisis químico del biodiésel obtenido con el catalizador heterogéneo en laboratorio y del biodiésel producido por Enremex S. de R.L. de C.V. Como puede apreciarse, la cantidad de ésteres metílicos es superior en el biodiésel obtenido con la zeolita. De la misma manera, la cantidad de ácidos grasos libres sin reaccionar es menor para el caso del catalizador heterogéneo, lo mismo que los mono y diglicéridos presentes en el producto final.

En los experimentos a nivel laboratorio se realizaron pruebas de reutilización de la zeolita en tres ciclos. El biodiésel obtenido presenta características fisicas y químicas muy similares. El rendimiento de biodiésel no varió más allá de $2 \%$ en cada ciclo. Por lo cual, se considera que la zeolita se puede reutilizar por varios ciclos.

Todo lo anterior implica que la zeolita favorece la calidad del biodiésel. Las características del producto final son mejores que con el KOH. Aunque las condiciones de reacción de laboratorio no han sido optimizadas, por lo que es posible obtener mejores resultados. Aunado a esto, la recuperación del catalizador heterogéneo y la limpieza del biodiésel son más fáciles de realizar.

También es importante mencionar que la desactivación de la zeolita será causada principalmente por la acumulación de carbono. Por lo tanto, es posible reutilizar el catalizador como suplemento en la formulación de alimento para animales o como componente de fertilizantes.

Por otro lado, es conveniente remarcar que la empresa productora de biodiésel sigue un plan de mejora continua en su proceso. En la actualidad, el producto se comercializa a bajo costo (aún competitivo) y el comprador lo mezcla con biodiésel obtenido a partir de aceites vírgenes para aumentar el contenido de ésteres metílicos. De la misma manera, se trabaja en la modificación de parámetros y pretratamientos que mejoren la calidad del biodiésel directamente en la producción.

En el cuadro II se presenta el inventario utilizado para la realización del presente estudio. Es importante señalar que el factor de emisión del aceite residual a cualquier categoría de impacto ambiental es

CUADRO I. CARACTERÍSTICAS DEL BIODIÉSEL OBTENIDO CON DIFERENTES CATALIZADORES

\begin{tabular}{lcccc}
\hline Muestra & Catalizador & $\begin{array}{c}\text { Ésteres metílicos } \\
(\%)\end{array}$ & $\begin{array}{c}\text { Ácidos grasos libres } \\
(\%)\end{array}$ & $\begin{array}{c}\text { Mono y diglicéridos } \\
(\%)\end{array}$ \\
\hline Biodiésel de grasa residual & Zeolita & 92.42 & 4.63 & 2.95 \\
Biodiésel Enremex S. de R.L. de C.V. & $\mathrm{KOH}$ & 89.66 & 5.86 & 4.48 \\
\hline
\end{tabular}


CUADRO II. INVENTARIO PARA ELANÁLISIS DEL CICLO DE VIDA DE LA PRODUCCIÓN DE BIODIÉSEL

\begin{tabular}{llccc}
\hline Parámetro & Etapa & Unidad & Escenario 1 & Escenario 2 \\
\hline Grasa residual & Transporte & $\mathrm{L}$ & 1000 & 1000 \\
Camión diésel & Transporte & $\mathrm{t}$ km & 2.5 & 2.5 \\
Rendimiento de camión & Transporte & $\mathrm{km} / \mathrm{L}$ & 3.5 & 3.5 \\
Recorrido en camión & Transporte & $\mathrm{km}$ & 2.5 & 2.5 \\
Camioneta gasolina & Transporte & $\mathrm{t} * \mathrm{~km}$ & 2 & 2 \\
Rendimiento de camioneta & Transporte & $\mathrm{km} / \mathrm{L}$ & 6.5 & 6.5 \\
Recorrido en camioneta & Transporte & $\mathrm{km}$ & 10 & 10 \\
KOH & Producción & $\mathrm{kg}$ & 9 & -- \\
Metanol & Producción & $\mathrm{L}$ & 208 & 795 \\
Electricidad & Producción & $\mathrm{MJ}$ & 86.4 & 86.4 \\
Agua & Producción & $\mathrm{L}$ & 900 & -- \\
Biodiésel producido & Producción & $\mathrm{L}$ & 900 & 1197 \\
Glicerol producido & Manejo residuos & $\mathrm{L}$ & 200 & 206 \\
Camión diésel & Manejo residuos & $\mathrm{t} * \mathrm{~km}$ & 8 & 8 \\
Recorrido en camión & Manejo residuos & $\mathrm{km}$ & 40 & 40 \\
Regulador de pH & Manejo residuos & $\mathrm{kg}$ & 20 & 10 \\
Camioneta gasolina & Manejo residuos & $\mathrm{t} * \mathrm{~km}$ & 1.1 & -- \\
Recorrido en camioneta & Manejo residuos & $\mathrm{km}$ & 15 & 15 \\
Zeolita & Producción & $\mathrm{kg}$ & -- & 26.1 \\
HCl (37\%) & Producción & $\mathrm{L}$ & -- & 1.218 \\
Agua destilada & Producción & $\mathrm{L}$ & -- & 298 \\
Metanol recuperado & Manejo residuos & $\mathrm{L}$ & -- & 264 \\
Residuos sólidos & Manejo residuos & $\mathrm{kg}$ & 73.7 & -- \\
\hline & & & & \\
\hline & & & & \\
\hline & & & & \\
\hline
\end{tabular}

0 (cero). Esto se debe a que en el proceso bajo estudio se considera la recuperación de un residuo para ser transformado en un producto de valor agregado.

\section{Evaluación de impactos del análisis}

El análisis se efectuó con el programa openLCA versión 1.6.3, el cual cuenta con una base de datos que se adapta a las necesidades del estudio y utiliza los procedimientos estandarizados para el procesamiento de datos.

Se utilizó el método de cálculo desarrollado por el Instituto de Ciencias Ambientales (CML, por sus siglas en holandés) de la Universidad de Leiden, Holanda (Guinée 2002), específicamente la versión marcada en el programa como "CML (baseline) [v4.4, January 2015]”. Se eligió este método debido a que permite determinar el impacto sobre las categorías de interés para la empresa.

Dada la existencia de un coproducto, se utilizó el método físico por masa para la asignación de impactos. Las categorías de impacto consideradas corresponden a la evaluación de potencial de:

- Agotamiento de recursos (combustibles fósiles)

- Toxicidad humana

- Ecotoxicidad
- Eutrofización

- Acidificación del agua

- Daño a la capa de ozono

- Cambio climático a 100 años

\section{RESULTADOS Y DISCUSIÓN}

\section{Impactos ambientales por escenario}

En las figuras 3 y 4 se encuentran los resultados del ACV para los dos escenarios. Se presenta la contribución de cada etapa al impacto total del escenario correspondiente.

En el cuadro III se muestran los valores numéricos de los impactos ambientales potenciales del proceso estudiado para cada escenario. Los datos están relacionados con la unidad funcional, es decir, corresponden al procesamiento de $1000 \mathrm{~L}$ de grasa residual.

En la figura 3 se incluyen los resultados para el escenario actual. Se observa que la etapa de producción tiene la mayor contribución en todas las categorías consideradas. Esto se debe principalmente a dos factores: el consumo de electricidad y la preparación del catalizador homogéneo (mezcla de hidróxido de potasio con metanol). 


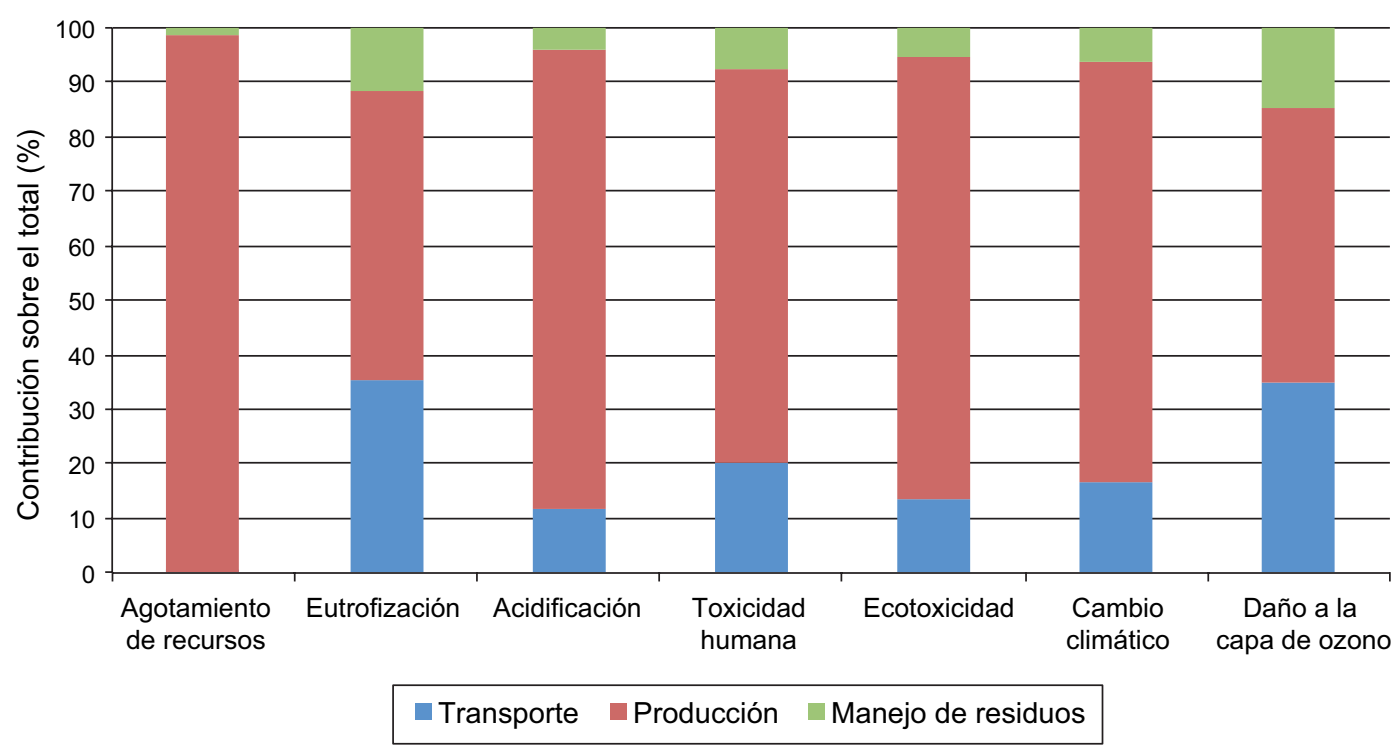

Fig. 3. Impactos del escenario 1: transesterificación con catalizador homogéneo

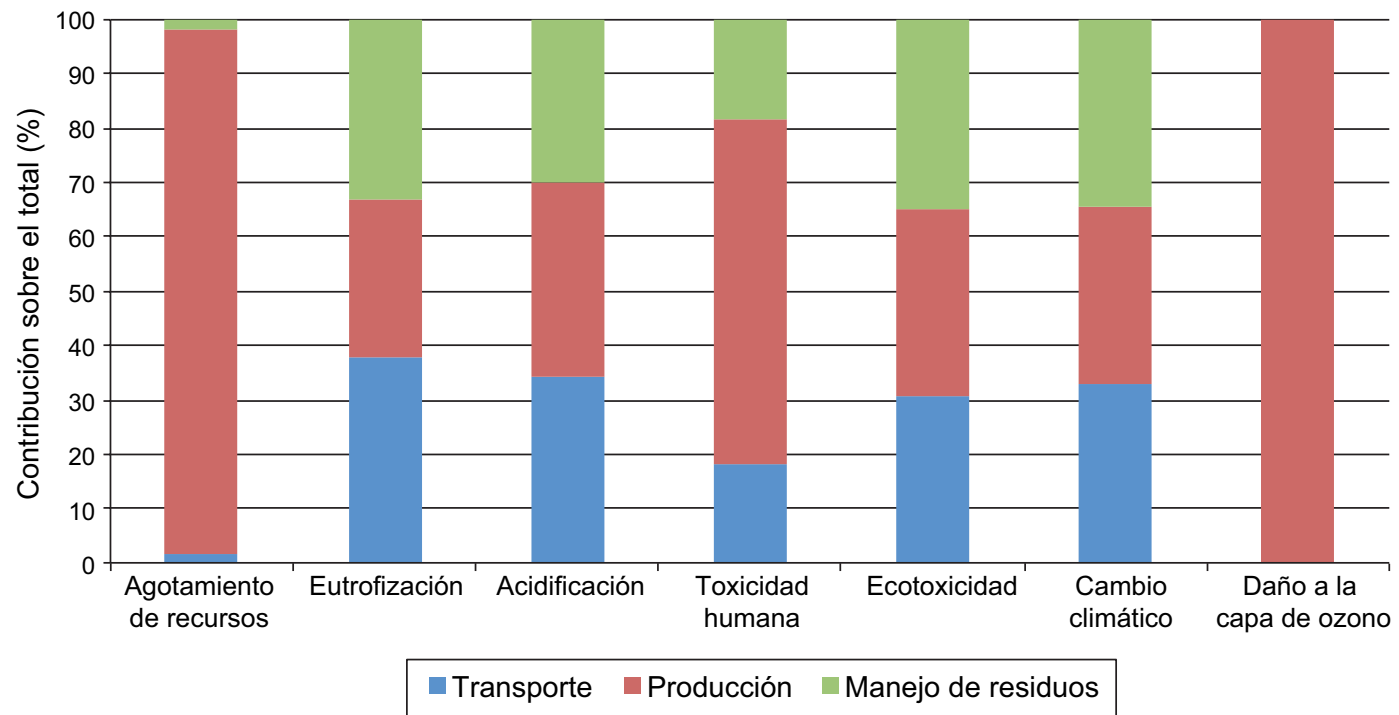

Fig. 4. Impactos del escenario 2: transesterificación con catalizador heterogéneo

CUADRO III. VALORES DE LOS IMPACTOS AMBIENTALES EN CADA ESCENARIO

\begin{tabular}{lccc}
\hline Categoría de impacto & Unidad & Escenario 1 & Escenario 2 \\
\hline Agotamiento de recursos & $\mathrm{MJ}$ & 390.39 & 25.700 \\
Eutrofización & $\mathrm{kg} \mathrm{PO}_{4}$ eq & 0.0212 & 0.0066 \\
Acidificación & $\mathrm{kg} \mathrm{SO}_{2}$ eq & 0.2423 & 0.0280 \\
Toxicidad humana & $\mathrm{kg} p$-DCB eq & 0.7190 & 0.2642 \\
Ecotoxicidad & $\mathrm{kg} p$-DCB eq & 0.2023 & 0.0292 \\
Cambio climático & $\mathrm{kg} \mathrm{CO}$ eq & 51.036 & 8.6640 \\
Daño a capa de ozono & $\mathrm{kg} \mathrm{CFC-11} \mathrm{eq}$ & $1.03 \times 10^{-11}$ & $2.46 \times 10^{-7}$ \\
\hline
\end{tabular}

$p$-DCB eq = equivalente químico de para-diclorobenceno, $\mathrm{CFC}$-11 eq = equivalente químico de clorofluorocarbono 11 
La electricidad de la región se produce a partir de combustibles fósiles y el metanol es un derivado del petróleo, lo cual se refleja en el agotamiento de los recursos.

Asimismo, los procesos involucrados en la obtención de estos insumos (electricidad, metanol y $\mathrm{KOH}$ ) provocan emisiones al aire, suelo y agua. Las primeras contribuyen al cambio climático por la liberación de gases con efecto invernadero. Los efectos de las emisiones al suelo y agua se observan a través de las categorías de eutrofización, acidificación y ecotoxicidad. De la misma manera, los tres tipos de emisiones contribuyen en la toxicidad humana.

Respecto al potencial de daño a la capa de ozono, es importante señalar que el escenario 1 emite apenas $1.03 \times 10^{-11}$ en kilogramos de equivalentes químicos de clorofluorocarbono 11 ( $\mathrm{kg}$ CFC11 eq). La etapa de producción contribuye con $50 \%$ y se debe principalmente al uso de derivados del petróleo.

Por su parte, el transporte contribuye de manera notoria en cinco de las siete categorías consideradas. Aunque los vehículos utilizan combustibles fósiles, su contribución al agotamiento de recursos es menor a $1 \%$. Esto se debe a que el consumo de combustible es muy poco en proporción con los recursos consumidos en la generación de electricidad y derivados del petróleo. No obstante, las emisiones de $\mathrm{CO}_{2}$ eq generadas por la combustión de diésel y gasolina contribuyen con $17 \%$ del potencial de cambio climático. La contribución en las otras categorías está asociada con el proceso de obtención de ambos combustibles a partir del petróleo.

La etapa de manejo de residuos es la que menor contribución tiene en el impacto ambiental. Esto se debe principalmente a que los volúmenes son bajos y a la neutralización de los residuos sólidos y líquidos. De esta manera, la mayor contribución proviene del desplazamiento de los residuos a sus lugares de venta o disposición.

Los resultados del análisis para el escenario 2 aparecen en la figura 4. Aunque la producción sigue siendo la que más contribuye al impacto total, el transporte y el manejo de residuos tienen una aportación mayor en cinco de las siete categorías. Esto se deriva de la sustitución del catalizador homogéneo por el heterogéneo.

La etapa de producción contribuye con $96 \%$ al agotamiento de recursos debido, otra vez, al consumo de electricidad y metanol. Además, el potencial de daño a la capa de ozono se atribuye completamente a esta etapa. La razón es que la zeolita requiere de un lavado con agua destilada, la cual pasa por procesos como purificación con luz ultravioleta y desozo- nización. Esto explica también el aumento en las emisiones de CFC11-eq, las cuales alcanzan un valor de $2.46 \times 10^{-7} \mathrm{~kg}$. La contribución de la producción en las otras cinco categorías se debe a las mismas razones expuestas para el escenario 1 .

El transporte, al igual que en el caso anterior, requiere de combustibles fósiles. La producción de éstos, así como su aplicación en motores de combustión interna contribuyen en las categorías de eutrofización, acidificación del agua, toxicidad humana, ecotoxicidad y cambio climático. El mayor porcentaje del transporte en estas cinco categorías se debe a que los valores numéricos de la producción se reducen drásticamente (un porcentaje mayor que el de las otras etapas).

En relación con el manejo de residuos, los volúmenes de producción de éstos son casi nulos. El principal residuo es el agua utilizada en el proceso de lavado de la zeolita. Su neutralización es una de las causas de la contribución de esta etapa en las diferentes categorías. La otra es el transporte del glicerol al lugar de venta.

Finalmente, en la figura 5 se muestra una comparación de los dos escenarios. En cada categoría se tomó el máximo valor como el $100 \%$, los porcentajes para los otros escenarios se calcularon en función de este valor máximo. Se observa que el escenario 1 tiene el mayor impacto en las primeras seis categorías. Existe una disminución notable en el escenario 2.

Como se ha indicado previamente, la disminución de los valores numéricos entre un escenario y otro se debe en gran medida al remplazo de insumos. Por ejemplo, se deja de utilizar hidróxido de potasio y se favorece la recuperación de metanol. Adicionalmente, el uso de la zeolita en más de un ciclo de producción reduce también el consumo de insumos. Además de lo anterior, de acuerdo con los datos presentados en el cuadro III, el proceso analizado (escenario 1) contribuye al agotamiento de recursos con un consumo de $390.39 \mathrm{MJ}$. Si se considera que el poder calorífico del biodiésel es alrededor de $35 \mathrm{MJ} / \mathrm{L}$, el proceso bajo estudio genera $31500 \mathrm{MJ}(900 \mathrm{~L}$ de biodiésel). La razón de energía (energía útil generada/energía fósil consumida) es de 80.68 , lo cual significa que se compensa el agotamiento de recursos.

De la misma manera, el aprovechamiento del biodiésel implicará beneficios ambientales, puesto que su combustión genera emisiones biogénicas. El análisis de esta y algunas otras situaciones se plantea ahora como parte de trabajos futuros, donde se amplíen los alcances del ACV.

La mayor contribución de la etapa de producción encontrada en este análisis concuerda con otros 


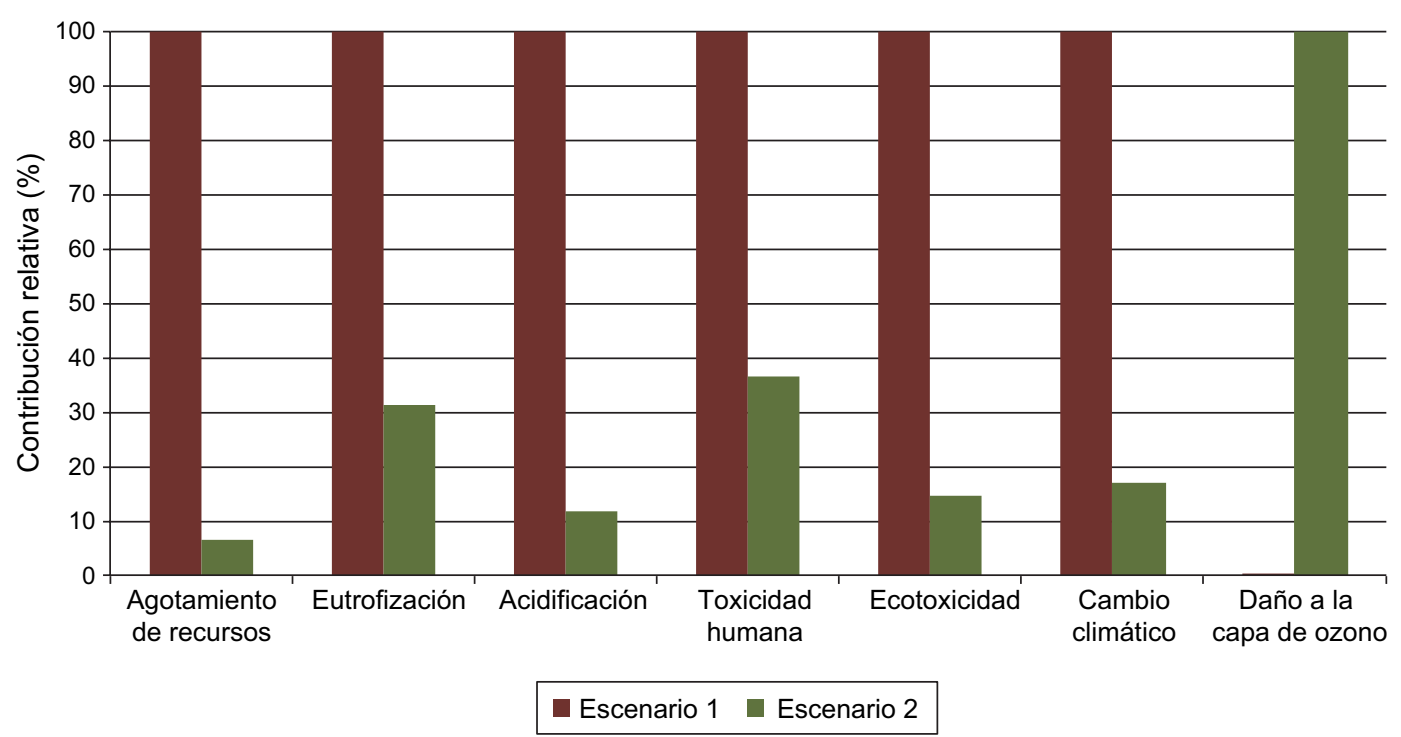

Fig. 5. Comparación de escenarios. Escenario $1=$ transesterificación con catalizador homogéneo. Escenario 2 $=$ transesterificación con catalizador heterogéneo

estudios (Talens Peiró et al. 2010, Caldeira et al. 2016), en los que se atribuye el uso de electricidad y metanol como las principales causas.

De la misma manera, hay coincidencia en cuanto a la contribución del catalizador a base de $\mathrm{KOH}$ con otras investigaciones (Lombardi et al. 2018).

Por otro lado, a diferencia de los resultados aquí expuestos, la etapa de recolección y transporte ha sido reportada como la que más contribuye (Caldeira et al. 2016, Siegel Moecke et al. 2016). Esto se debe principalmente a las mayores distancias consideradas en tales estudios.

\section{Distribución de impactos ambientales}

La distribución de impactos totales entre los productos obtenidos se presenta en la figura 6.

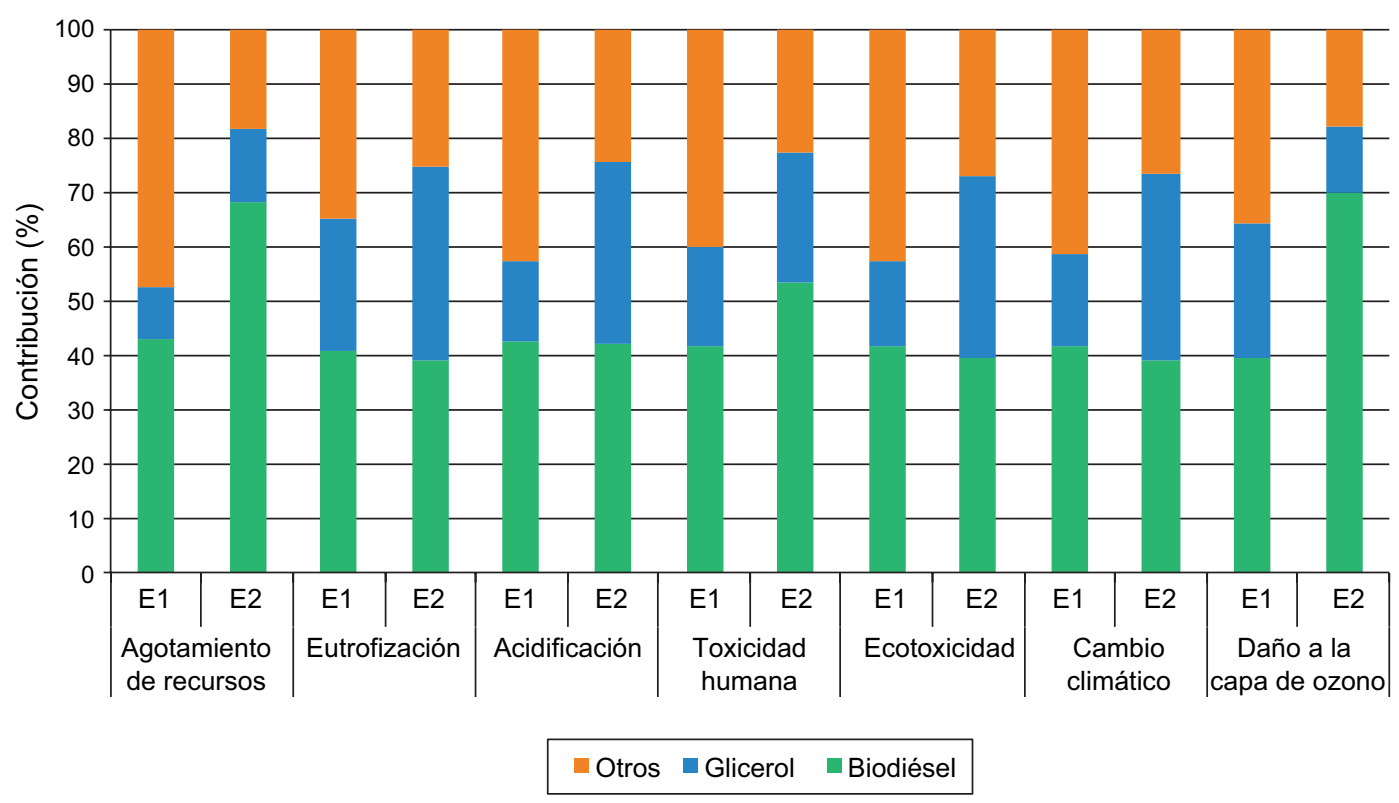

Fig. 6. Distribución de impactos ambientales en función de productos y subproductos. $\mathrm{E}=$ escenario. Escenario $1(\mathrm{E} 1)=$ transesterificación con catalizador homogéneo. Escenario $2(\mathrm{E} 2)=$ transesterificación con catalizador heterogéneo 
Para el escenario 1, alrededor de $40 \%$ corresponde al biodiésel en todas las categorías evaluadas. Esto se debe a que la asignación de impactos se realizó mediante un método físico considerando la masa de los diferentes flujos. Asimismo, el biodiésel se obtiene en la etapa que presenta los mayores impactos ambientales. Por otro lado, el glicerol es el subproducto con menor asignación de impactos debido a que presenta la menor cantidad de masa respecto a los otros productos.

En el escenario 2, los impactos asignados al biodiésel aumentan en las categorías de agotamiento de recursos, toxicidad humana y daño a la capa de ozono. Esta situación está asociada con el uso de agua destilada en la preparación del catalizador heterogéneo. Por su parte, el porcentaje asignado al glicerol se incrementa en cuatro de las categorías consideradas, esto se debe también en gran medida al uso de agua destilada en el proceso de producción. En el caso de los otros residuos, tienen una asignación menor de impactos en todas las categorías porque su masa ha disminuido gracias al uso del catalizador heterogéneo.

\section{Identificación de áreas de oportunidad}

Aún cuando en este trabajo no se realizó una comparación con otros combustibles (diésel fósil, biodiésel de cultivos energéticos, biodiésel de algas), es de esperar que el biodiésel de aceites residuales provea ventajas ambientales. En este sentido, los resultados obtenidos pueden utilizarse como base para realizar un análisis comparativo con otros combustibles.

Respecto al diésel fósil, se evita la emisión de gases de efecto invernadero de origen fósil y se contribuye a la reducción del consumo de recursos. En cuanto a otros tipos de biodiésel, se evita la competencia alimenticia y también la del uso de suelo. Además, se extiende la cadena productiva de los aceites vegetales, propiciando una producción más limpia.

Así, es interesante conocer la contribución potencial del biodiésel de aceites residuales en el cumplimiento de las metas de reducción de gases con efecto invernadero, establecidas por entidades internacionales de certificación, tales como la Directiva Europea de Energía Renovable (EU-RED) o la Mesa Redonda sobre Biocombustibles Sustentables (SBR, por sus siglas en inglés). Para ello, se plantea como un trabajo futuro realizar un estudio adicional relativo a la producción de aceites residuales en el país, así como evaluar las emisiones del biocombustible en la etapa de aprovechamiento (combustión).
Por otro lado, se identifican las siguientes oportunidades para mejorar el desempeño ambiental del proceso de producción de biodiésel:

- Modificar la etapa de lavado de la zeolita para reducir el potencial de daño a la capa de ozono. Es necesario minimizar o eliminar el uso de agua destilada para una aplicación en la empresa. Se requiere una corriente de agua con bajo contenido de sales para evitar una eventual desactivación de la zeolita. Así, se propone evaluar el uso de agua purificada o de agua residual tratada con baja conductividad.

- Evaluar otros mecanismos de revaloración de glicerol y compararlos con el propuesto en este trabajo. Por ejemplo, producir metanol para sustituir este insumo que actualmente es de origen fósil.

- Evaluar otras fuentes de energía renovable, tales como la energía solar, para generar el calor requerido en la etapa de producción.

Finalmente, cabe señalar que para tomar decisiones en torno a las acciones para mejorar el desempeño del proceso, es necesario realizar un análisis tecnoeconómico de la transesterificación heterogénea. Esto permitirá complementar los resultados del análisis ambiental.

\section{CONCLUSIONES}

En este trabajo se realizó el ACV de un proceso de producción de biodiésel a partir de aceites residuales vegetales. Se planteó el uso de un catalizador heterogéneo como alternativa al catalizador actualmente en uso por la empresa productora.

Se encontró que la etapa de producción es la que más contribuye al impacto ambiental. El escenario 1 presenta la mayor influencia en todas las categorías consideradas. Esto se debe al consumo de electricidad y derivados del petróleo.

Asimismo, la etapa de transporte contribuye con más de $30 \%$ en el potencial de daño a la capa de ozono y eutrofización, con menos de $20 \%$ en las categorías de acidificación del agua, toxicidad humana, ecotoxicidad y cambio climático. La razón de esta situación es el uso de combustibles fósiles, los cuales generan emisiones al aire, agua y suelo. Por su parte, la etapa de manejo de residuos es la que menos contribuye al impacto en todas las categorías, lo cual es una consecuencia del transporte a los sitios de disposición y a la neutralización de los residuos. 
En este sentido, la aplicación del catalizador heterogéneo representa ventajas importantes: disminuye el uso de insumos, facilita la separación de productos y subproductos y reduce la generación de residuos. Esto se refleja en una menor emisión de contaminantes: entre $65 \%$ y $95 \%$ menos que para el caso del escenario 1. Lo anterior mejora en general el desempeño ambiental del proceso. Sólo el potencial de daño a la capa de ozono es mayor, lo cual es una consecuencia del uso de agua destilada.

\section{AGRADECIMIENTOS}

Los autores agradecen al personal de la empresa Enremex S. de R.L. de C.V. por facilitar las muestras de grasas residuales y por la información operativa para la elaboración de este estudio. También se agradece al Consejo Nacional de Ciencia y Tecnología (CONACYT) de México por el financiamiento para la realización de este proyecto (PEI 199274).

\section{REFERENCIAS}

Caldeira C., Queirós J., Noshadravan A. y Freire F. (2016). Incorporating uncertainty in the life cycle assessment of biodiesel from waste cooking oil addressing different collection systems. Resour. Conserv. Recy. 112, 83-92. DOI: 10.1016/j.resconrec.2016.05.005

Curran M.A. (2014) Strengths and limitations of life cycle assessment En Background and future prospects in life cycle assessment (W. Klöpffer, Ed.). Springer, Dordrecht, Holanda, pp. 189-206. DOI: 10.1007/978-94-017-8697-3_6

Finnveden G., Hauschild M.Z., Ekvall T., Guinée J., Heijungs R., Hellweg S., Koehler A., Pennington D. y Suh. S. (2009). Recent developments in life cycle assessment. J. Environ. Manage. 91 (1), 1-21.

DOI: 10.1016/j.jenvman.2009.06.018

Guinée J.B. (2002). Handbook on life cycle assessment. Kluwer Academic Publishers. Dordrecht, Holanda, 692 pp. DOI: $10.1007 / 0-306-48055-7$

Guldbrandssonn F. y Bergmark P. (2012). Opportunities and limitations of using life cycle assessment methodology in the ICT Sector. Memorias. Electronics Goes 2012+. Berlin, Alemania. 9 al 12 de septiembre, pp. 1-6.

Hajjari M., Tabatabaei M., Aghbashlo M. y Ghanavati H. (2017). A review on the prospects of sustainable biodiesel production: A global scenario with an emphasis on waste-oil biodiesel utilization. Renew. Sust. Ener. Rev. 72, 445-464. DOI: 10.1016/j.rser.2017.01.034
ISO 14040 (2006). Norma ISO 14040. Environmental management - Life cycle assessment - Principles and framework. Organización Internacional de Normalización. Ginebra, Suiza.

ISO 14044 (2006). Norma ISO 14044. Environmental management - Life cycle assessment - Requirements and guidelines. Organización Internacional de Normalización. Ginebra, Suiza.

Lombardi L., Mendecka B. y Carnevale E. (2018). Comparative life cycle assessment of alternative strategies for energy recovery from used cooking oil. J. Environ. Manage. 216, 235-245.

DOI: 10.1016/j.jenvman.2017.05.016

Mandolesi de Araújo C.D., de Andrade C.C., de Souza e Silva E. y Dupas F.A. (2013). Biodiesel production from used cooking oil: A review. Renew. Sust. Ener. Rev. 27, 445-452. DOI: 10.1016/j.rser.2013.06.014

Muralikrishna I.V. y Manickam V. (2017). Life cycle assesment. En: Environmental management (I.V. Muralikrishna y V. Manickam, Eds.). Elsevier, Oxford, Reino Unido, pp. 57-75.

DOI: 10.1016/B978-0-12-811989-1.00005-1

SEMARNAT (2017). Ley general del equilibrio ecológico y la protección al ambiente. Secretaría de Medio Ambiente, Recursos Naturales y Pesca. Diario Oficial de la Federación. 24 de enero de 2017.

Siegel Moecke E.H., Feller R., dos Santos H.A., de Medeiros Machado M., Vieira Cubas A.L., de Aguiar Dutra A.R., Vieira Santos L.L. y Roberto Soares S. (2016). Biodiesel production from waste cooking oil for use as fuel in artisanal fishing boats: integrating environmental, economic and social aspects. J. Clean. Prod. 135, 679-688. DOI: 10.1016/j.jclepro.2016.05.167

Tacias Pascasio V.G., Rosales Quintero R. y Torrestiana Sánchez B. (2016). Evaluación y caracterización de grasas y aceites residuales de cocina para la producción de biodiésel: un caso de estudio. Rev. Int. Contam. Ambie. 32 (3), 303-313.

DOI: 10.20937/RICA.2016.32.03.05

Talebian-Kiakalaieh A., Saidina Amin NA. y Mazaheri H. (2013). A review on novel processes of biodiesel production from waste cooking oil. Appl. Ener. 104, 683-710. DOI: 10.1016/j.apenergy.2012.11.061

Talens Peiró L., Lombardi L., Villalba Méndez G. y Gabarrell i Durany X. (2010). Life cycle assessment (LCA) and exergetic life cycle assessment (ELCA) of the production of biodiesel from used cooking oil (UCO). Energy 35 (2), 889-893.

DOI: $10.1016 /$ j.energy.2009.07.013

Wallace T., Gibbons D., O’Dwyer M. y Curran T.P. (2017). International evolution of fat, oil and grease (FOG) waste management - A review. J. Environ. Manage. 187, 424-435. DOI: 10.1016/j.jenvman.2016.11.003 
Yaakob Z., Mohammada M., Alherbawi M., Alam Z. y Sopian K. (2013). Overview of the production of biodiesel from waste cooking oil. Renew. Sust. Ener. Rev. 18, 184-193.

DOI: 10.1016/j.rser.2012.10.016
Yano J., Aoki T., Nakamura K., Yamada K. y Sakai Sh-i. (2015). Life cycle assessment of hydrogenated biodiesel production from waste cooking oil using the catalytic cracking and hydrogenation method. Waste Manage. 38, 409-423. DOI: 10.1016/j.wasman.2015.01.014 


\section{ANEXO. DESCRIPCIÓN DE UN EXPERIMENTO DE CATÁLISIS HETEROGÉNEA}

A continuación se describe el procedimiento utilizado para la preparación del catalizador heterogéneo, así como para la obtención de biodiésel a partir de grasas residuales vegetales.

\section{Preparación del catalizador}

1. Tamizado. La zeolita se tamizó con una malla para obtener fracciones de partículas de malla $7-8$ (2.38 $\mathrm{mm}-2.83 \mathrm{~mm}$ )

2. Desaluminación. Este paso consiste en remover total o parcialmente los átomos de aluminio del esqueleto cristalino de la zeolita y con esto aumentar el acceso a la porosidad del catalizador. Se realizó un tratamiento ácido con una solución de $\mathrm{HCl} 0.1 \mathrm{~N}$ y $3 \mathrm{~N}(200 \mathrm{~mL})$. La zeolita se colocó en dos columnas de intercambio iónico, por las cuales se hizo circular la solución ácida a una velocidad de 20 gotas $/ \mathrm{min}$.

3. Lavado. El lavado tiene por objetivo eliminar impurezas, tales como materia orgánica y algunos otros materiales. En un matraz de $1 \mathrm{~L}$ se colocó $50 \%$ en volumen de zeolita desaluminada y $50 \%$ de agua destilada $(200 \mathrm{~mL})$. La mezcla se mantuvo en agitación durante $30 \mathrm{~min}$ a temperatura ambiente. Posteriormente se dejó secar la zeolita a $100^{\circ} \mathrm{C}$ durante $72 \mathrm{~h}$ en un horno de laboratorio para eliminar completamente la humedad.

\section{Tratamiento de la materia prima}

4. Secado. Las muestras de grasa vegetal residual se calentaron a $100{ }^{\circ} \mathrm{C}$ para eliminar el contenido de agua.

5. Separación de partículas. Las muestras se centrifugaron a una velocidad y durante un tiempo que permitieron eliminar impurezas y obtener homogeneidad.

\section{Obtención de biodiésel}

6. Transesterificación. Se realizó en un matraz de fondo redondo de tres bocas con capacidad de $500 \mathrm{~mL}$ equipado con un refrigerante a reflujo. Se consideró una relación molar metanol:grasa de 6:1. Se colocaron $100 \mathrm{~mL}$ de grasa residual en el matraz y se calentó en baño María. Al alcanzar $50^{\circ} \mathrm{C}$ en el interior, se encendió el agitador y se adicionó lentamente la mezcla de catalizador con metanol, la cual fue previamente calentada a la temperatura de reacción. Este conjunto de sustancias se calentó durante $2 \mathrm{~h}$ con agitación constante.

7. Separación de productos. Una vez concluido el tiempo de reacción, la mezcla se transfirió a un embudo de decantación y se mantuvo en reposo para la separación de productos. Se separó el biodiésel, se lavó con agua caliente a $80{ }^{\circ} \mathrm{C}$ (aproximadamente un $40 \%$ respecto a la cantidad de aceite) hasta alcanzar un pH neutro.

8. Análisis de productos. El biodiésel obtenido se analizó por cromatografía de gases acoplada a espectrometría de masas para determinar su calidad y obtener conclusiones respecto al procedimiento utilizado. 